\title{
Inhalt
}

Ludwig M. Eichinger

Praktiken: etwas Gewissheit im Geflecht der alltäglichen Welt — VII

Arnulf Deppermann/Helmuth Feilke/Angelika Linke

Sprachliche und kommunikative Praktiken: Eine Annäherung

aus linguistischer Sicht 1

\section{Praktiken der leiblich-verbalen Interaktion}

Margret Selting

Praktiken des Sprechens und Interagierens im Gespräch aus der Sicht von Konversationsanalyse und Interaktionaler Linguistik — 27

Jürgen Streeck

Gestische Praxis und sprachliche Form — 57

Anja Stukenbrock

Deiktische Praktiken: Zwischen Interaktion und Grammatik — 81

Stephan Habscheid

Handeln in Praxis. Hinter- und Untergründe situierter

sprachlicher Bedeutungskonstitution -127

Wolfgang Imo

Im Zweifel für den Zweifel: Praktiken des Zweifelns — 153

Susanne Günthner/Katharina König

Kommunikative Gattungen in der Interaktion:

Kulturelle und grammatische Praktiken im Gebrauch — 177

Axel Schmidt

Am Rande der Praktik - Körperliche Eigendynamiken und ihre Funktionalisierung am Beispiel von Reality-TV — 205

Hubert Knoblauch/René Tuma

Praxis, kommunikatives Handeln und die Videoanalyse

der Videoanalyse -229 
VI Inhalt

\section{Textuelle Praktiken}

Helmuth Feilke

Literale Praktiken und literale Kompetenz — 253

Michael Beißwenger

Praktiken in der internetbasierten Kommunikation — 279

Joachim Scharloth

Praktiken modellieren: Dialogmodellierung als Methode

der Interaktionalen Linguistik — 311

Jannis Androutsopoulos

Mediatisierte Praktiken: Zur Rekontextualisierung von

Anschlusskommunikation in den Sozialen Medien - 337

Juliane Schröter

Vom Handeln zur Kultur. Das Konzept der Praktik in der Analyse von Verabschiedungen 369

Doris Tophinke

„In den tiefsten Winkeln unserer Betonwälder tanzten die Namen

ein farbenfrohes Fest und wir tanzten mit bis in die Morgenstunden" Zur praktischen Kultur des Szene-Graffiti — 405

Daniel Perrin

Vom vielschichtigen Planen. Textproduktions-Praxis empirisch erforscht -431

Thomas Gloning

Neue mediale Formate und ihre kommunikative Nutzung in der Wissenschaft. Fallbeispiele und sieben Thesen zum

Praktiken-Konzept, seiner Reichweite und seinen Konkurrenten 
Ludwig M. Eichinger (Mannheim)

\section{Praktiken:}

\section{etwas Gewissheit im Geflecht der alltäglichen Welt}

Die Sprache muss für sich selber sprechen. (Ludwig Wittgenstein: Philosophische Grammatik, § 27)

\section{Sprachlich und kommunikativ}

\subsection{Allgemeines}

Das Thema der diesmaligen Jahrestagung „Sprachliche und kommunikative Praktiken“ lässt sicherlich, wie die meisten Themen, die mittels des Konnektors und verbunden sind, verschiedene Lesarten zu. Auf jeden Fall spannen die beiden Adjektive dieser Fügung ein Feld auf, bei dem man neugierig ist, wie das Verhältnis der Elemente sprachlich und kommunikativ im Einzelnen aufgelöst werden wird. ${ }^{1}$ Aber es geht bei dieser Art der Formulierung unausweichlich um Praktiken auch im Bezug auf Sprache, also nicht unmittelbar um etwas wie Systemstrukturen. Eigentlich spricht das Reden von Praktiken von den Grenzen dessen, was eine systemlinguistische Abstraktion leisten kann. Bei solch einer Fokussierung ist die rein systemlinguistische Bestandsaufnahme eher eine Voraussetzung für die Behandlung des Praktiken-Themas. Das Funktionieren der sprachlichen Regelhaftigkeiten ist eine der Grundlagen, von deren Funktionieren man im Prinzip ausgeht, an deren Funktionalität auch kein vernünftiger Zweifel besteht, wenn man über Praktiken einigermaßen sinnvoll reden will.

1 Wovon die Beschreibung der Funktion (wenn auch der Propositionen verknüpfenden) des Konnektors und in Breindl/Volodina/Waßner (2014, S. 394 f. und 407 ff.) beredtes Zeugnis gibt. 


\section{2 sprachlich}

Denn natürlich ist es wichtig, zu wissen, wie die Strukturen der Sprache funktionieren, wenn man sehen und bewerten will, wie uns die Sprache erlaubt, in der Welt mit ihren Herausforderungen angemessen zu agieren. Die Idee der Einbettung in den Rahmen situationsbezogenen Funktionierens bringt allerdings auch bei der Beschreibung der sprachlichen Mittel eine Fokussierung mit sich, die es erlaubt, die Fragen der systematischen Ausstattung unter den Bedingungen dieses Vorkommens zu bewerten. Es lässt sich über die Akzeptabilität oder so etwas wie die systemgerechte Wohlgeformtheit und ihre Beschreibung in der gesprochenen Sprache ohne ein Wissen über die Bandbreite der Praktiken nicht so recht reden. Nun ist in den letzten Jahrzehnten viel dazu geforscht worden, was die medial bzw. konzeptionell bedingten Spezifika gesprochener Sprache ausmacht. ${ }^{2}$ Über eine Bestandsaufnahme spezifischer Formen und Strukturen hinaus, die in manchen Bereichen durchaus noch weiterer empirischer Fundierung bedürfte, geht es dabei nicht zuletzt darum, in der Beschreibung umzusetzen, was die spezifischen Verarbeitungsbedingungen gesprochener Sprache betrifft. Ausgehend von den Bedingungen von Dialogizität ${ }^{3}$ ergibt sich eine Ausgestaltung des sprachlichen Teils von Praktiken, deren Gestalt und Umfang sich aus der Interaktion mit den anderen Möglichkeiten situativer Orientierung, Information, Anweisung oder Ausdrucksintention ergibt. Dieser Tatbestand reduziert im Normalfall den Grad an Explizitheit, den wir von skriptural konzipierten Texten erwarten, gerade auch, weil in medialen Kontexten, die von Erwartungen geprägt sind, die wir mit Oralität verbinden, ein höherer Grad an Explizierung und damit auch Explizitheit nachgefordert werden kann. ${ }^{4}$

2 Auch mit einer langen Tradition am IDS (siehe schon Steger 1988), zusammenfassend Schwitalla (2012); vielfach aufbauend auf Modellen, die zwischen der formalen und einer funktionalen Ebene unterscheiden: Oralität/Skripturalität (Koch/Österreicher 1985); Nähe/Distanz (Ágel/Hennig (Hg.) 2010); Operator/Operand (Fiehler et al. 2004). Sie haben auch ihren Weg in die Standarddarstellungen gefunden (siehe Duden 2009).

$3 \mathrm{Zu}$ deren insgesamt grundlegendem Status, der sich etwa in pronominalen Systemen niederschlägt, siehe etwa Weinrich (2004, S. 17 f.) oder Hagège (1987).

4 Dass hier auch praktisch-deiktische Explizierungen möglich sind, soll an dieser Stelle nicht weiter thematisiert werden. Zu entsprechenden Phänomenen im Oralitäts-Skripturalitäts-Übergangsbereich vgl. die Ausführungen zu Ellipsen in Zifonun/Hoffmann/Strecker (1997, S. 409-442; Verf.: Ludger Hoffmann). 


\title{
1.3 kommunikativ
}

Für die Kenntnis und Beschreibung von Praktiken kommt aber noch dazu, dass Muster von Situationen mit ihren „regelhaften“ sprachlichen Elementen identifiziert werden sollen. Das heißt, es geht darum, wie bestimmte Strukturen mit interaktionalen Herausforderungen und Wendungen verbunden werden. Im Hinblick auf die Interpretation sprachlicher Formen in solchen Kontexten passt das zu Überlegungen, wie sie Ludwig Wittgenstein in „Über Gewissheit“ zur Verwendung bestimmter sprachlicher Formen anstellt: ${ }^{5}$

\begin{abstract}
Wenn dies aber nicht bloß eine Bemerkung der deutschen Grammatik sein soll, so muss er (= der Philosoph/L.E.) die Umstände angeben, unter denen dieser Ausdruck funktioniert. (Ludwig Wittgenstein: Über Gewissheit, § 433)
\end{abstract}

Man kann zweifellos verschiedene Vorstellungen davon entwickeln, was es heißen kann, die Umstände anzugeben, unter denen ein Ausdruck funktioniert. Ganz offenkundig aber kann es nicht um individuelle Festlegungen gehen, wenn es sich um gelingende Züge eines Sprachspiels handelt. Von hier lässt sich über einen weiteren Schritt die Verbindung zu der typischen Struktur des Sprachlichen im Rahmen von Praktiken herstellen. Praktiken funktionieren ja nur, wenn sie als Muster, also wiederkehrende aber variable Konstellationen und Prozessformate erkennbar sind. Auf die strukturierende Wirkung solcher musterhafter Einheiten muss man sich verlassen können, wenn Interaktionen möglichst unaufwändig ablaufen sollen. Es ist offenkundig, dass die Verlässlichkeit, mit der wir sprachliche Äußerungen als konstituierende Elemente von gesellschaftlichen Übereinkünften zu Praktiken des Handelns lesen können, eben nicht nur an der Sprache festzumachen sind, den aus ihr inferierbaren Behauptungen und den daraus gezogenen Schlüssen über geteilte Überzeugungen und dergleichen. ${ }^{6}$ Vielmehr, um noch einmal Wittgensteins „Über Gewissheit“ zu zitieren, kann man sagen:

\footnotetext{
5 Dabei akzentuiert die Vorstellung des „Sprachspiels“, die Wittgenstein entwickelt, kurz gesprochen, Fragen einer Gebrauchsbedeutung (siehe etwa Harras 2004, S. 103) und liegt somit auf einer anderen Ebene als das Konzept der Praktik - sie lässt sich aber in diesem Kontext gut verstehen. Vgl. dazu auch Wittgenstein (2001, S. 956 und 1060).

6 Auch wenn man auf sprachlicher Ebene - und nicht nur bei gesprochener Sprache - eine solche Ebene der Strukturierung feststellen kann, vgl. dazu Eichinger (2010, S. 50, 2014).
} 
Unsre Rede erhält durch unsere übrigen Handlungen ihren Sinn (Ludwig Wittgenstein: Über Gewissheit, § 229)

Die „Rede“ Wittgensteins, also die sprachlichen Bestandteile von geteilten Handlungsmustern können ihre Intentionalität erst im Kontext der Umstände normativer Zusammenhänge erkennen lassen. ${ }^{7}$ Diese Objekte der sozialen Welt geben uns die in der alltäglichen Kommunikation normalerweise nicht hinterfragte Gewissheit, die uns unauffällig sprachlich handeln lässt (ober eben vielleicht auch bewusst nicht). Und auch andersherum gilt, dass die Frage, welche Funktion bestimmte Äußerungstypen haben, erst in diesen Kontexten angemessen beantwortet werden kann. So geht es darum, aus den Praktiken der Handhabung durch gemeinsame Wahrnehmung geprägter Abläufe die Funktionalität bestimmter sprachlicher Einsätze zu beurteilen. Die Besonderheit des sprachlichen Teils der Praktiken dürfte es allerdings sein, dass die sprachliche Kodierung in der Lage ist, der im Zweifel aufkommenden Pflicht zum Explizitmachen in komplexen Zusammenhängen effektiv nachzukommen - und eigentlich fast beliebig explizit zu sein. Im Normalfall braucht es aber im Alltag genau das nicht, sondern einen an der Verlässlichkeit der Interaktion orientierten angemessenen (mäßigen) Gebrauch der sprachlichen Mittel.

\section{4 verlässlich}

Die Verlässlichkeit des sprachlichen Handelns ohne ständige explizite Begründung funktioniert auf der Annahme der Realität und Gültigkeit geteilter Handlungsmuster. Es sind das die - auch sprachlichen - Muster, in die wir hineinwachsen. ${ }^{8}$ Wenn sie Praktiken des Alltags sind, brauchen sie nicht die dauernde Begründung, vielmehr liefern sie, ohne dass wir ständig daran zweifelten, eine Vorstellung davon, was (in gewissen intentionalen Kontexten) ein erwartbares und akzeptables Verhalten ist. ${ }^{9}$ Sie sind damit ein Teil und Objekt unserer Reali-

7 So passt die Diskussion um Praktiken gut zu der derzeit in der Philosophie aktuellen Auseinandersetzung um einen „Neuen Realismus“; vgl. die Beiträge in Markus (Hg.) (2014).

8 Unterschiedliche Aspekte dieser Sichtweise werden z.B. in Linke (2011) oder Feilke (2003) thematisiert.

9 Natürlich ist die Gewissheit über die Regularitäten in den jeweiligen Mustern nicht unbegrenzt; so kann man bestimmte sprachliche Mittel als die Aufforderung verstehen, gegebenenfalls Explizitheit einzuklagen (oder eben Einverständnis mit der Interpretation der Situation zu signalisieren), etwa Abtönungspartikeln des Deutschen, die auf gemeinsame Vorannahmen verweisen. 
tät. ${ }^{10}$ Aber natürlich sind solche Übereinkünfte auch nichts, was sich nicht verändern ließe oder verändern würde. Gerade Rekurrenz und Variation der gewählten (sprachlichen) Ausdrucksformen erlauben es, das, was sich uns als ein multimodaler ${ }^{11}$ Strom der Interaktion darbietet, analytisch zu zerteilen, und so die Sprache - zudem die gesprochene - als Untersuchungsobjekt in ihrem natürlichen Habitat, in der Welt der intentionalen Praktiken funktional und formal zu beschreiben. ${ }^{12}$

\section{5 wozu}

Betrachtung der Sprache kann, wie am Anfang festgestellt, die verschiedensten Dinge betreffen und die verschiedensten Wege gehen. Von der Orientierung an Praktiken, wie sie in der hier dokumentierten 51. Jahrestagung des IDS an verschiedensten Objekten und unter verschiedensten methodischen und theoretischen Blickwinkeln betrieben wird, kann man sich dreierlei versprechen:

- Zum einen erlaubt sie eine feingranulare und auf Variation angelegte Beschreibung sprachlicher Formen in actu.

- Zum zweiten lassen sich die situationellen Bedingungen als Bestandteile eines Experiments nutzen und modifizieren; das dient der Überprüfbarkeit der Aussagen über die Prägungskraft und relative Festigkeit von Praktiken.

- Zum dritten ist der Blick aus der sprachwissenschaftlichen Welt auf das Konzept der Praktiken ein Ernstnehmen einer sozialwissenschaftlichen Interpretation sprachlichen Verhaltens als Bestandteil von sozialen Handlungsmustern.

Was das konkret heißt, und was die theoretischen und empirischen Felder sind, die behandelt wurden, wird in der folgenden fachlichen Einführung dargelegt. ${ }^{13}$

10 Gerade die Möglichkeit, zu handeln und dabei durch Implizitlassen zu „sparen“, spricht davon, dass es sich hier nicht um eine immer wiederholte Konstruktion, sondern eine auf festgewordene Erfahrung sich stützende Realität handelt (siehe dazu Markus (Hg.) 2014 - übrigens auch Phänomene, die gegenüber geschriebener Sprache wie redundant aussehen).

11 Das ist vielleicht der Punkt, um darauf hinzuweisen, dass die Umbrüche im kommunikativen Leben, die durch die IT-basierten Medien bedingt sind, neue Arten kommunikativer Praktiken hervorbringen.

$12 \mathrm{Zu}$ einer entsprechenden Konzeption von Pragmatik siehe z.B. Deppermann (2015, bes. S. 330 und 347).

13 Die Verfasser dieser Einführung, Arnulf Deppermann, Helmuth Feilke und Angelika Linke, haben gemeinsam auch die Tagung inhaltlich vorbereitet. Ihnen sei hier dafür gedankt. 


\section{Darüber hinaus}

Die Tagung des Jahres 2015 stand zudem wieder im Lichte der Verleihung des Konrad-Duden-Preises der Stadt Mannheim. ${ }^{14}$ Er wurde auf einem Festakt im Rahmen der Jahrestagung an Damaris Nübling verliehen.

\section{Literatur}

Ágel, Vilmos/Hennig, Mathilde (Hg.) (2010): Nähe und Distanz im Kontext variationslinguistischer Forschung. (= Linguistik - Impulse und Tendenzen 35). Berlin/New York.

Breindl, Eva/Volodina, Anna/Waßner, Ulrich Hermann (2014): Handbuch der deutschen Konnektoren 2: Semantik der deutschen Satzverknüpfer. 2 Teilbde. (= Schriften des Instituts für Deutsche Sprache 13). Berlin/Boston.

Deppermann, Arnulf (2015): Pragmatik revisited. In: Eichinger, Ludwig M. (Hg.): Sprachwissenschaft im Fokus. Positionsbestimmungen und Perspektiven. (= Jahrbuch des Instituts für Deutsche Sprache 2014). Berlin/Boston, S. 323-352.

Duden (2009): Der Duden in 12 Bänden: Bd. 4: Die Grammatik. 8., überarb. Aufl. Mannheim u.a.: Dudenverlag.

Eichinger, Ludwig M. (2010): Der durchschnittliche Linguist und die Daten. Eine Annäherung. In: Kratochvílová, Iva/Wolf, Norbert Richard (Hg.): Kompendium Korpuslinguistik. Eine Bestandsaufnahme aus deutsch-tschechischer Perspektive. (= Germanistische Bibliothek 38). Heidelberg: Winter, S. 27-51.

Eichinger, Ludwig M. (2014): Was man braucht, kann nicht fehlen. Grammatik, Textstil und Interaktionsmodalität. In: Kolehmainen, Leena/Tiittula, Liisa/Lenk, Hartmut (Hg.): Kommunikative Routinen. Formen, Formeln, Forschungsbereiche. Festschrift zum 65. Geburtstag von Irma Hyvarinen. Frankfurt a.M. u.a., S. 153-167.

Feilke, Helmuth (2003): Textroutine, Textsemantik und sprachliches Wissen. In: Linke, Angelika/ Ortner, Hanspeter/Portmann Paul R. (Hg.): Sprache und mehr. Ansichten einer Linguistik der sprachlichen Praxis. (= Reihe Germanistische Linguistik 245). Tübingen, S. 209-229.

Fiehler, Reinhard et al. (2004): Eigenschaften gesprochener Sprache. Theoretische und empirische Untersuchungen zur Spezifik mündlicher Kommunikation. (= Studien zur Deutschen Sprache 30). Tübingen.

Hagège, Claude (1987): Der dialogische Mensch. Sprache - Weltbild - Gesellschaft. Reinbek: Rowohlt.

14 Der Stadt Mannheim und dem Dudenverlag als der Verleiherin und dem finanziellen Ausstatter dieses Preises sei dafür gedankt, mit diesem Preis die Möglichkeit geschaffen zu haben, herausragende Arbeit im Bereich der deutschen Sprachwissenschaft in bemerkenswerter Weise zu würdigen. Frau Bürgermeisterin Dr. Ulrike Freundlieb hat in ihrem Grußwort nicht nur die Verbundenheit des IDS mit der Stadt bestätigt, sondern in so konkreter wie überzeugender Weise gezeigt, wie die Frage geteilter oder divergierender Praktiken ein Thema ist, das eine moderne städtische Gesellschaft durchaus angeht. 
Harras, Gisela (2004): Handlungssprache und Sprechhandlung. Eine Einführung in die theoretischen Grundlagen. 2. erw. u. durchges. Aufl. Berlin/New York.

Koch, Peter/Österreicher, Wulf (1985): Sprache der Nähe - Sprache der Distanz. Mündlichkeit und Schriftlichkeit im Spannungsfeld von Sprachtheorie und Sprachgeschichte. In: Romanistisches Jahrbuch 36, S. 15-43.

Linke, Angelika (2011): Politics as linguistic performance: Function and 'Magic' of communicative practices. In: Steinmetz, Willibald (Hg.): Political languages in the age of extremes. Oxford, S. 53-66.

Markus, Gabriel (Hg.) (2014): Der neue Realismus. Berlin.

Schwitalla, Johannes (2012): Gesprochenes Deutsch. 4. Aufl. (= Grundlagen der Germanistik 33). Berlin.

Steger, Hugo (1988): Erscheinungsformen der deutschen Sprache. ,Alltagssprache' - ,Fachsprache' - ,Standardsprache' - ,Dialekt' und andere Gliederungstermini. In: Deutsche Sprache 16, S. 289-319.

Weinrich, Harald (2004): Textgrammatik der deutschen Sprache. 3. Aufl. Hildesheim.

Wittgenstein, Ludwig (1969): Philosophische Grammatik. (= Schriften 4). Frankfurt a.M.

Wittgenstein, Ludwig (1970): Über Gewissheit. Frankfurt a.M.

Wittgenstein, Ludwig (2001): Philosophische Untersuchungen. Frankfurt a.M.

Zifonun, Gisela/Hoffmann, Ludger/Strecker, Bruno (1997): Grammatik der deutschen Sprache. (= Schriften des Instituts für Deutsche Sprache 7). Berlin/New York. 
